\title{
How Health Care Workers Wield Influence Through Twitter Hashtags: Retrospective Cross-sectional Study of the Gun Violence and COVID-19 Public Health Crises
}

Ayotomiwa Ojo $^{1 *}$, BS; Sharath Chandra Guntuku ${ }^{2,3,4^{*}}, \mathrm{PhD}$; Margaret Zheng ${ }^{4,5}$, BA; Rinad S Beidas ${ }^{5}$, PhD; Megan L Ranney ${ }^{6}, \mathrm{MD}, \mathrm{MPH}$

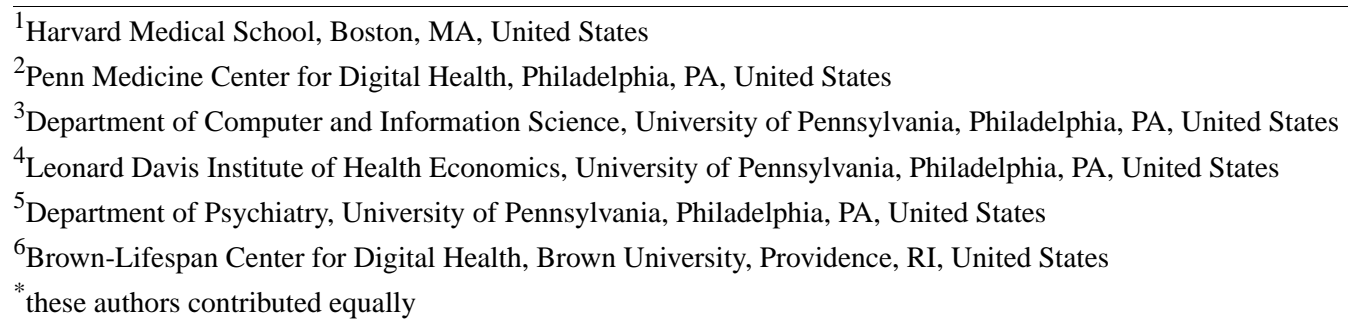

Corresponding Author:

Megan L Ranney, MD, MPH

Brown-Lifespan Center for Digital Health

Brown University

139 Point St

Providence, RI, 02903

United States

Phone: 16466443053

Email: megan ranney@brown.edu

\section{Abstract}

Background: Twitter has emerged as a novel way for physicians to share ideas and advocate for policy change. \#ThisIsOurLane (firearm injury) and \#GetUsPPE (COVID-19) are examples of nationwide health care-led Twitter campaigns that went viral. Health care-initiated Twitter hashtags regarding major public health topics have gained national attention, but their content has not been systematically examined.

Objective: We hypothesized that Twitter discourse on two epidemics (firearm injury and COVID-19) would differ between tweets with health care-initiated hashtags (\#ThisIsOurLane and \#GetUsPPE) versus those with non-health care-initiated hashtags (\#GunViolence and \#COVID19).

Methods: Using natural language processing, we compared content, affect, and authorship of a random $1 \%$ of tweets using \#ThisIsOurLane (Nov 2018-Oct 2019) and \#GetUsPPE (March-May 2020), compared to \#GunViolence and \#COVID19 tweets, respectively. We extracted the relative frequency of single words and phrases and created two sets of features: (1) an open-vocabulary feature set to create 50 data-driven-determined word clusters to evaluate the content of tweets; and (2) a closed-vocabulary feature for psycholinguistic categorization among case and comparator tweets. In accordance with conventional linguistic analysis, we used a $P<.001$, after adjusting for multiple comparisons using the Bonferroni correction, to identify potentially meaningful correlations between language features and outcomes.

Results: In total, 67\% ( $\mathrm{n}=4828)$ of \#ThisIsOurLane tweets and 36.6\% ( $\mathrm{n}=7907)$ of \#GetUsPPE tweets were authored by health care professionals, compared to $16 \%(n=1152)$ of \#GunViolence and 9.8\% $(n=2117)$ of \#COVID19 tweets. Tweets using \#ThisIsOurLane and \#GetUsPPE were more likely to contain health care-specific language; more language denoting positive emotions, affiliation, and group identity; and more action-oriented content compared to tweets with \#GunViolence or \#COVID19, respectively.

Conclusions: Tweets with health care-led hashtags expressed more positivity and more action-oriented language than the comparison hashtags. As social media is increasingly used for news discourse, public education, and grassroots organizing, the public health community can take advantage of social media's broad reach to amplify truthful, actionable messages around public health issues. 
(JMIR Public Health Surveill 2021;7(1):e24562) doi: 10.2196/24562

\section{KEYWORDS}

COVID-19; firearm injury; social media; online advocacy; Twitter; infodemiology; infoveillance; tweet; campaign; health care worker; influence; public health; crisis; policy

\section{Introduction}

Twitter has emerged as a novel way for physicians to organize and advocate for policy change, and combat misinformation amid national health crises. One in 5 adults in the United States uses Twitter, and $75 \%$ report using this platform as a news outlet [1]. When Twitter advocacy campaigns brand their movement with a hashtag, tagged tweets are easily archived and found, opening up discussions to users who do not have any personal connection to the authors.

\#ThisIsOurLane and \#GetUsPPE are examples of health care-initiated Twitter movements that went viral. In November 2018, in response to the National Rifle Association's tweet asserting that "Someone should tell self-important anti-gun doctors to stay in their lane...," Dr Michael Gonzalez coined \#ThisIsOurLane to describe why health care professionals are involved in firearm injury prevention and treatment [2]. During the COVID-19 pandemic, Dr Esther Choo initiated \#GetMePPE, later expanded to \#GetUsPPE, to raise awareness about critical personal protective equipment (PPE) shortages [3]. Anecdotes suggest \#ThisIsOurLane influenced societal perceptions of health care professionals' role in firearm injury [4], and \#GetUsPPE galvanized attention to hospitals' unmet PPE needs $[5,6]$.

Whether online discussions with health care-initiated hashtags actually differ from contemporaneous discussions of the firearm injury and COVID-19 epidemics has not been quantified. Nor, to our knowledge, has the involvement of Twitter users outside of health care been examined. Understanding the content and voice of health professionals on social media during public health crises is essential. Rampant misinformation about health care online has led to international debates about how best to change public knowledge and conversations. At the same time, some experts are bemoaning "infodemics," in which people are so overwhelmed by contradictory facts that they become unable to act to protect themselves and their families [7]. Examining the content, tone, and types of tweeters involved in health care-led social media campaigns could inform future efforts related to data dissemination by the medical and nonmedical community [8].

To examine the characteristics of these online discussions, we compared psycholinguistic characteristics (ie, content and affect) of tweets among two cohorts: contemporaneous tweets regarding gun violence (comparing tweets with \#ThisIsOurLane vs \#GunViolence) and contemporaneous tweets regarding the COVID-19 pandemic (\#GetUsPPE/\#GetMePPE vs \#COVID19). We hypothesized that messages using health care-led hashtags would be more negative in tone (reflecting frustration and negative directives) but also more actionable in content (providing solutions) compared with non-health care-related hashtags, given health care professionals' personal stake and proximity to these issues.

\section{Methods}

This retrospective cross-sectional study selected a random $1 \%$ sample of publicly available Twitter data containing specific hashtags from across the United States.

\section{Data}

For cohort 1, we identified tweets containing \#ThisIsOurLane $(\mathrm{n}=38,774)$ or \#GunViolence $(\mathrm{n}=52,183)$ between November 7, 2018, and October 13, 2019, given multiple episodes of gun violence with national attention during this time period. For cohort 2, we identified tweets with \#GetUsPPE or \#GetMePPE $(\mathrm{n}=39,658)$ or \#COVID19 $(\mathrm{n}=200,000)$ between March 17, 2020, and May 20, 2020, which reflects the duration of the campaign at the time of the analysis. Both study periods began when the hashtag was introduced. After discarding retweets and tweets containing only hashtags and user mentions (without any other words), 7201 \#ThisIsOurLane tweets and 21,605 \#GetUsPPE/\#GetMePPE tweets remained as "cases". Tweets containing both case and control hashtags were preserved as cases in the analysis. A random sample of 7201 of the remaining \#GunViolence-only tweets and 21,605 of the \#COVID19-only tweets were selected as comparators for two separate analyses (Figure 1). Although tweets about gun violence and COVID-19 used other hashtags, these were identified as trending and potentially the most common around the study period and were used as comparators.

We used the Python package TwitterMySQL [9], which utilizes the official Twitter application programming interface (API), to collect tweets matching at least one of the keywords described above in real time. We note that the Twitter API limits such streams to $1 \%$ of the total Twitter volume at any given moment. Similar methods have been used in prior work studying health-related tweets [10-14].

We obtained Twitter profile descriptions of the users in our data set using the Twitter API and searched for words indicating health care professional status using regular expressions (eg, "doc*," "medic*," "surg*"). When processing tweets for this analysis, we only utilized information publicly available on users' Twitter profiles. The University of Pennsylvania Institutional Review Board deemed the study exempt. 
Figure 1. Study flowchart. API: application programming interface.

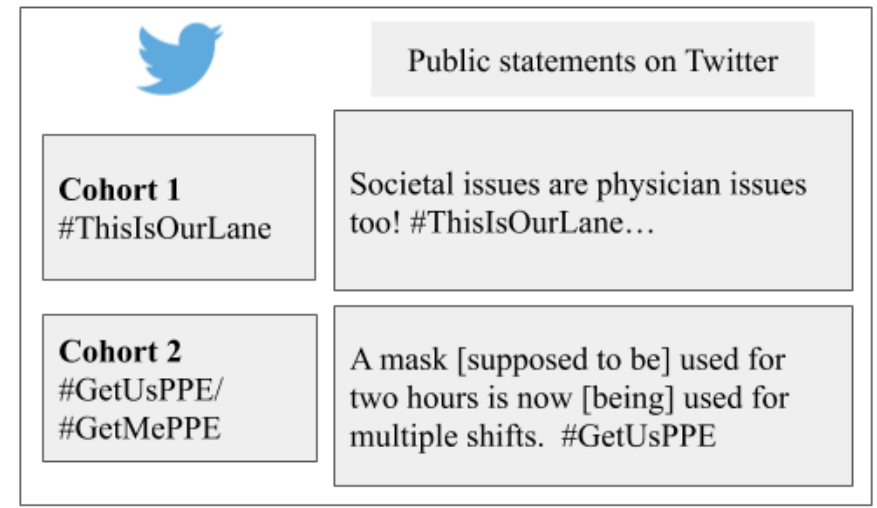

\section{Extracting Language Features}

After tokenizing the tweets [15], we extracted the relative frequency of single words and phrases and created two sets of features: (1) an open vocabulary feature set [16] defined using the MALLET (Machine Learning for Language Toolkit) implementation of latent Dirichlet allocation [17], an unsupervised clustering algorithm, to create 50 data-driven-determined word clusters; and (2) a closed vocabulary feature set defined as the normalized frequency of 71 psycholinguistic categories among case and comparator tweets, created with Linguistic Inquiry Word Count dictionary [18].

\section{Statistical Analysis}

Each feature set was input in a logistic regression model, with "case" (ie, \#ThisIsOurLane or \#GetUsPPE) as the dependent variable. In accordance with conventional linguistic analysis, we used a $P$ value of $<.001$, after adjusting for multiple comparisons using the Bonferroni correction, to identify potentially meaningful correlations between language features and outcomes. We calculated regression coefficients with the \#GunViolence and \#COVID19 (comparator) groups as references.

\section{Results}

In total, $67 \%(\mathrm{n}=4828)$ of \#ThisIsMyLane tweets and $36.6 \%$ $(n=7907)$ of \#GetUsPPE tweets were authored by health care
Downloaded tweets containing the hashtags using Twitter Streaming API.

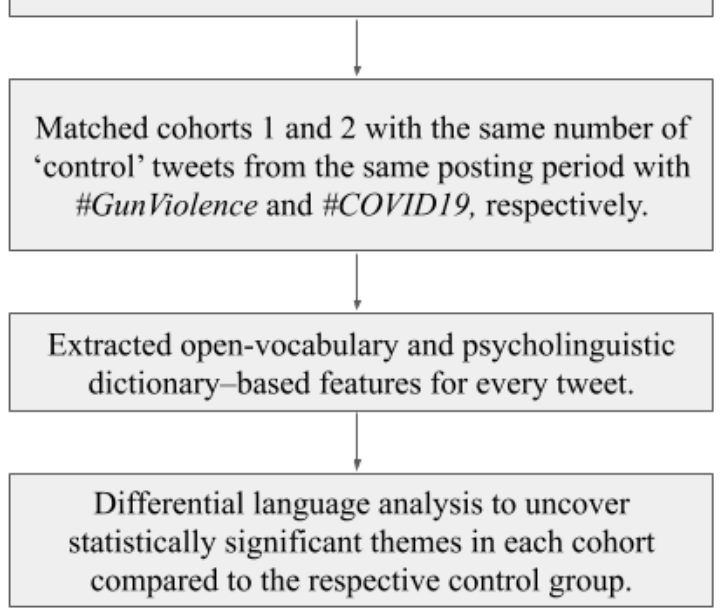

professionals, compared to $16 \%(\mathrm{n}=1152)$ of \#GunViolence and $9.8 \%(n=2117)$ of \#COVID19 tweets.

The open-vocabulary feature set (ie, content) of \#ThisIsOurLane and \#GetUsPPE were more likely to contain language specific to health care than general tweets using hashtags \#GunViolence and \#COVID19 (Figures 2-5). Specifically, \#ThisIsOurLane tweets discussed health care professionals' advocacy, research, or appreciation of colleagues, and were more likely to mention public health and community compared with \#GunViolence tweets. \#ThisIsOurLane tweets were less likely to mention political entities like \#NRA and specific events such as \#ElPaso. \#GetUsPPE tweets described severe PPE shortages for health care workers, the need to support patient and staff safety, and referenced health care workers as heroes. Additionally, \#GetUsPPE tweets included more action-oriented language (ie, deliver, sign, support) compared with \#COVID19 tweets.

Analysis of closed-vocabulary associations (ie, psycholinguistic categories) demonstrated that tweets with \#ThisIsOurLane or \#GetUsPPE contained more language associated with health, positive emotions, affiliation, and group identity compared to tweets with \#GunViolence or \#COVID19, respectively (Figure 6). General tweets about gun violence and the COVID-19 pandemic contained more words associated with negative emotions or anger than tweets with health care-initiated hashtags. 
Figure 2. Words associated with \#ThisIsOurLane tweets compared to \#GunViolence. Beta indicates the strength of association of each word with respective groups and color indicates frequency. All words are statistically significant at $\mathrm{p}<.05$, Benjamin Hochberg correction.

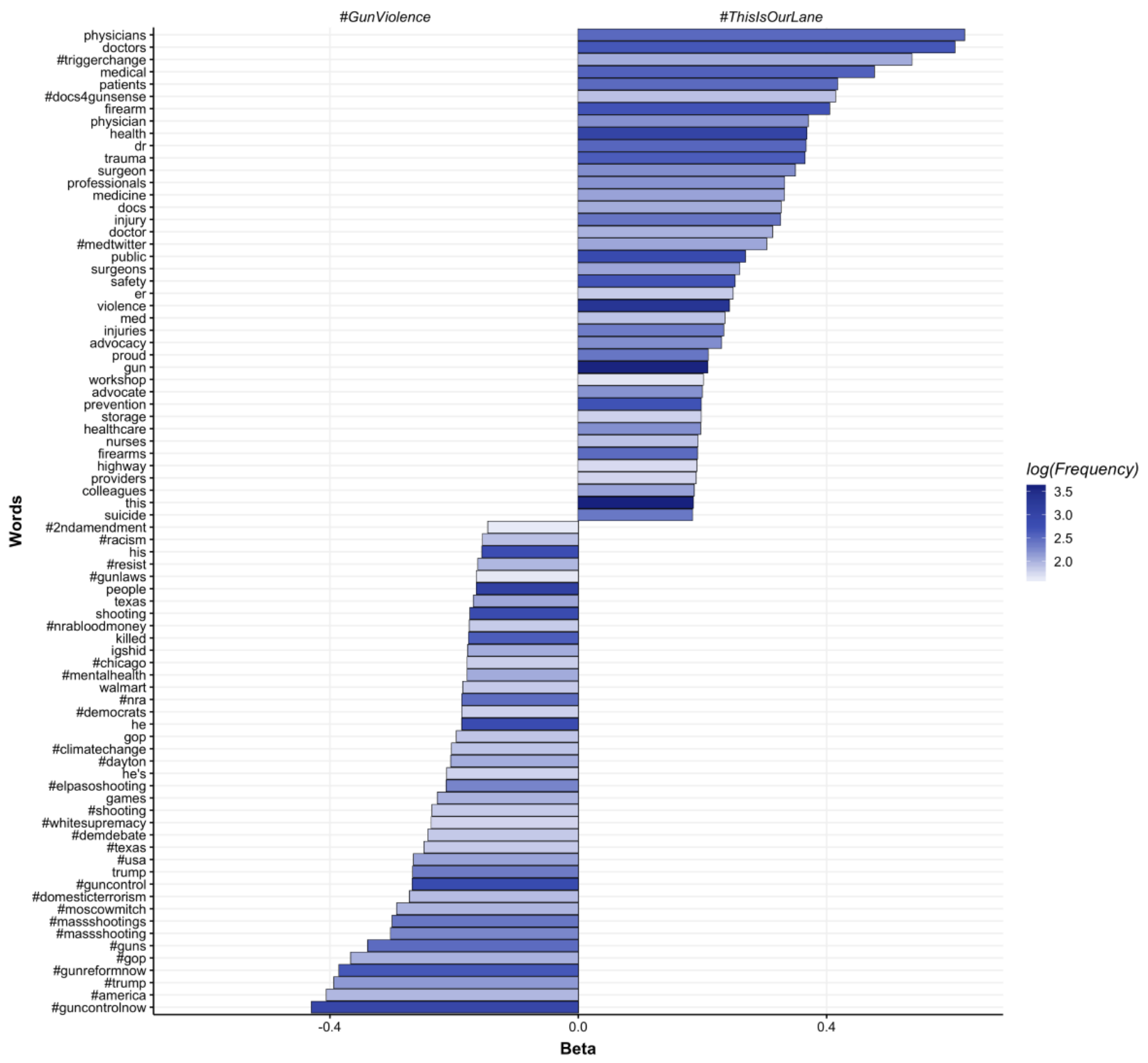


Figure 3. Words associated with \#GetUsPPE tweets compared to \#COVID19. Beta indicates the strength of association of each word with respective groups and color indicates frequency. All words are statistically significant at $\mathrm{p}<.05$, Benjamin Hochberg correction.

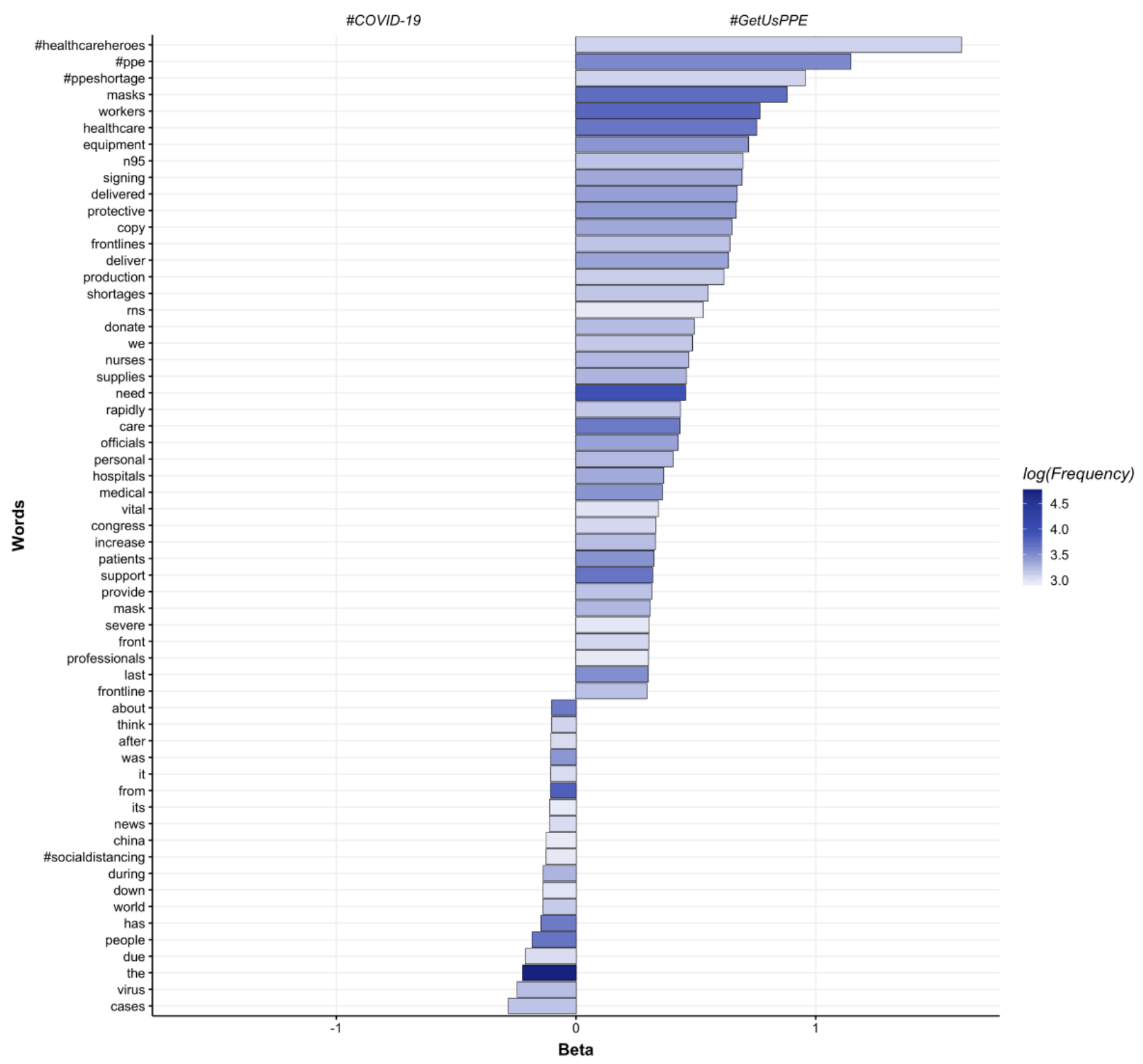


Figure 4. Highly correlated topics with mention of \#ThisIsOurLane vs. \#GunViolence. Beta indicates the strength of association of each topic. Top words and example paraphrased tweets for each topic are shown. Topics are statistically significant at $\mathrm{p}<.05$, Benjamin Hochberg correction.

\begin{tabular}{|c|c|c|c|}
\hline Topic Theme & Highly Correlated Words in the Topic & $\begin{array}{c}\text { Beta (>0: \#ThisisOurLane; <0: } \\
\text { \#GunViolence) }\end{array}$ & Example Tweets \\
\hline \multicolumn{4}{|c|}{ \#This/sOurlane } \\
\hline Medical Profession & $\begin{array}{l}\text { trauma, surgeon, surgeons, victims, gunshot, } \\
\text { patients, doctors, hospital, wounds, dr }\end{array}$ & 0.819 & $\begin{array}{l}\text { Emergency room doc sharing gut-wrenching stories of docs and their } \\
\text { experiences dealing with gun violence. \#ThislsOurLane }\end{array}$ \\
\hline Advocacy & $\begin{array}{l}\text { health, patients, care, medical, physicians, } \\
\text { \#medtwitter, advocacy, medicine, advocate, social }\end{array}$ & 0.773 & $\begin{array}{c}\text { "Doctors also have a \#professional duty to advocate on broader issues } \\
\text { affecting health, including tobacco, alcohol, poverty, and many other } \\
\text { issues" \#ThislsOurLane \#medtwitter <URL> }\end{array}$ \\
\hline Prevention & $\begin{array}{l}\text { firearm, injury, prevention, dr, research, death, } \\
\text { safety, injuries, prevent, deaths }\end{array}$ & 0.764 & $\begin{array}{l}\text { \#ThisIsOURLane American College of Surgeons recommendations for } \\
\text { reducing gun injury, death and disability. <URL> }\end{array}$ \\
\hline Appreciation & $\begin{array}{l}\text { proud, work, great, important, colleagues, stand, } \\
\text { working, leadership, story, powerful }\end{array}$ & 0.692 & $\begin{array}{l}\text { Proud of <USER > \& other \#emergencymedicine colleagues for } \\
\text { representing \#ThisIsOurLane \#publichealth \#BanAssaultWeapons <URL> }\end{array}$ \\
\hline Research & $\begin{array}{l}\text { research, violence, funding, safety, national, public, } \\
\text { approach, data, policy, prevention }\end{array}$ & 0.402 & $\begin{array}{l}\text { Yes. AND demand a long term comprehensive public health approach } \\
\text { to reducing gun violence. Support the creation of a National Bureau for } \\
\text { Gun Safety. \#thisisourlane }\langle\text { USER }>\langle\text { URL }>\end{array}$ \\
\hline \multicolumn{4}{|c|}{ \#GunViolence } \\
\hline \multirow[t]{3}{*}{ Specific Events } & $\begin{array}{l}\text { \#guncontrolnow, \#elpasoshooting, \#elpaso, } \\
\text { \#gunreformnow, trump, america, \#dayton, } \\
\text { \#daytonshooting, \#gunreform, } \\
\text { \#whitesupremacistterrorism }\end{array}$ & -1.097 & $\begin{array}{l}\text { I am sick of hearing about thoughts and prayers. \#gunreformnow } \\
\text { \#EIPasoShooting \#GunReform \#NRACarnage \#NRA } \\
\text { \#NRAIsATerroristOrganization \#walmartshooting \#EPShooting \#EIPaso } \\
\text { \#GunControlNow \#GunViolence }\end{array}$ \\
\hline & $\begin{array}{l}\text { mass, shootings, shooting, paso, el, dayton, } \\
\text { \#massshooting, texas, 2019, \#elpaso }\end{array}$ & -0.387 & $\begin{array}{l}\text { In addition to El Paso and Dayton, Virginia and Chicago also recorded } \\
\text { mass shootings this weekend: GunDeaths ... \#gunviolence }\end{array}$ \\
\hline & $\begin{array}{l}\text { shooting, people, killed, police, shot, dead, } \\
\text { injured, chicago, city, man }\end{array}$ & -0.413 & $\begin{array}{c}7 \text { wounded, } 1 \text { killed Wednesday in shootings across Chicago <URL> ... } \\
\text { \#EnoughisEnough \#StopChicagoShootings \#gunviolence } \\
\text { \#BlackTwitterMovement }\end{array}$ \\
\hline Political Affiliation & $\begin{array}{l}\text { vote, \# gunreformnow, put, care, nra, money, } \\
\text { protect, \#gunreform, republicans, americans }\end{array}$ & -0.685 & $\begin{array}{l}\text { I weep today for the victims lost due to \#GunViolence I weep for the } \\
\text { families. I weep for our country and for the soulless GOP that perfer to } \\
\text { line their pockets with money rather than put forth \#GunControlNow } \\
\text { \#mitchthemurderer \#TrumpsTerrorists \#massshootings }\end{array}$ \\
\hline Advocacy & $\begin{array}{l}\text { \#guncontrol, \#guncontrolnow, \#guns, } \\
\text { \#gunsense, \#nra, \# gunreform, \#2a, } \\
\text { \#gunreformnow, \#gun, \#usa }\end{array}$ & -1.14 & $\begin{array}{c}\text { >2.5 million lives are saved each year thru defensive gun use } \\
\text { by citizens. \#EnforceTheLaw \#Walkaway \#SecondAmendment } \\
\text { \#Selfdefense \#Rights \#2A \#NotAboutGuns \#GunReform } \\
\text { \#GunSense \#GunControl \#GunViolence \#GunControlNow } \\
\text { \#GunBan \#GOP \#NRA \#2AShallNotBelnfringed <URL> }\end{array}$ \\
\hline
\end{tabular}

Figure 5. Highly correlated topics with mention of \#GetUsPPE vs. \#COVID19. Beta indicates the strength of association of each topic. Top words and example paraphrased tweets for each topic are shown. Topics are statistically significant at $p<.05$, Benjamin Hochberg correction.

\begin{tabular}{|c|c|c|c|}
\hline Topic Theme & Highly Correlated Words in the Topic & $\begin{array}{l}\text { Beta (>0: \#GetUsPPE; <0: } \\
\text { \#COVID19) }\end{array}$ & Example Tweets \\
\hline \multicolumn{4}{|c|}{ \#GetUsPPE } \\
\hline \multirow[t]{2}{*}{ Advocacy } & $\begin{array}{l}\text { provide, work, patients, frontlines, care, congress, } \\
\text { shortages, severe, vital, experiencing }\end{array}$ & 1.603 & $\begin{array}{l}\text { RNs have been experiencing severe shortages in personal protective } \\
\text { equipment (PPE) as they work on the frontlines to provide vital care to } \\
\text { COVID-19 patients. Tell your member of Congress to do everything } \\
\text { possible to \#GetMePPE <URL> }\end{array}$ \\
\hline & $\begin{array}{l}\text { support, mass, delivered, deliver, II, officials, } \\
\text { increase, signing, copy, rapidly }\end{array}$ & 1.068 & $\begin{array}{l}\text { Support <USER> by signing "We Need to Rapidly Increase PPE Mass } \\
\text { Production" }\end{array}$ \\
\hline Support & $\begin{array}{l}\text { donate, supplies, medical, hospitals, masks, local, } \\
\text { share, supply }\end{array}$ & 1.35 & Donate medical supplies.\#GetMePPE <URL $>$ \\
\hline $\begin{array}{l}\text { Patient/Healthcare } \\
\text { Worker Safety }\end{array}$ & equipment, protective, personal, healthcare, lives & 1.315 & $\begin{array}{c}<\text { USER }><\text { USER > Our Health Care Workers Begging For Personal } \\
\text { Protective Equipment!\#GetMePPE <URL> }\end{array}$ \\
\hline Supply Shortages & $\begin{array}{l}\text { \#ppe, \#ppeshortage, make, masks, hospitals, } \\
\text { supplies, n95 }\end{array}$ & 1.295 & $\begin{array}{l}\text { \#GetUsPPE \#PPEshortage \#GetUsPPE \#PPEshortage THESE HEROS } \\
\text { NEED OUR HELP <URL> }\end{array}$ \\
\hline \multicolumn{4}{|c|}{ \#COVID19 } \\
\hline Political Affiliation & trump, virus, response, china & -0.101 & Trump is a Failure. \#impeachTrumpnow \#VoteDemocratic \\
\hline \multirow[t]{3}{*}{ General Discourse } & news, good, great, world, due, read & -0.198 & Some good news in the time of \#CoronaVirus \\
\hline & stop, spread, testing, virus, social, news & -0.229 & Please stop coming into restaurants \\
\hline & cases, testing, today, day, china & -0.319 & $\begin{array}{l}\text { Estimated to be over 100k cases and } 5 \text { confirmed cases of \#coronavirus } \\
\text { in \#Ohio. Geez }\end{array}$ \\
\hline Nation Association & virus, china, world, fight, due, it's, spread & -0.347 & It originated in China. It's a China virus. Grow up! \\
\hline
\end{tabular}


Figure 6. Linguistic correlates of health care-led Twitter hashtag campaigns (\#ThisIsOurLane and \#GetUsPPE/\#GetMePPE) compared with general ones (\#GunViolence and \#COVID19). Positive beta indicates a strong correlation of the linguistic category with the case compared to the control tweets. *"Power" was not significant at $P<.001$ for cohort 1.

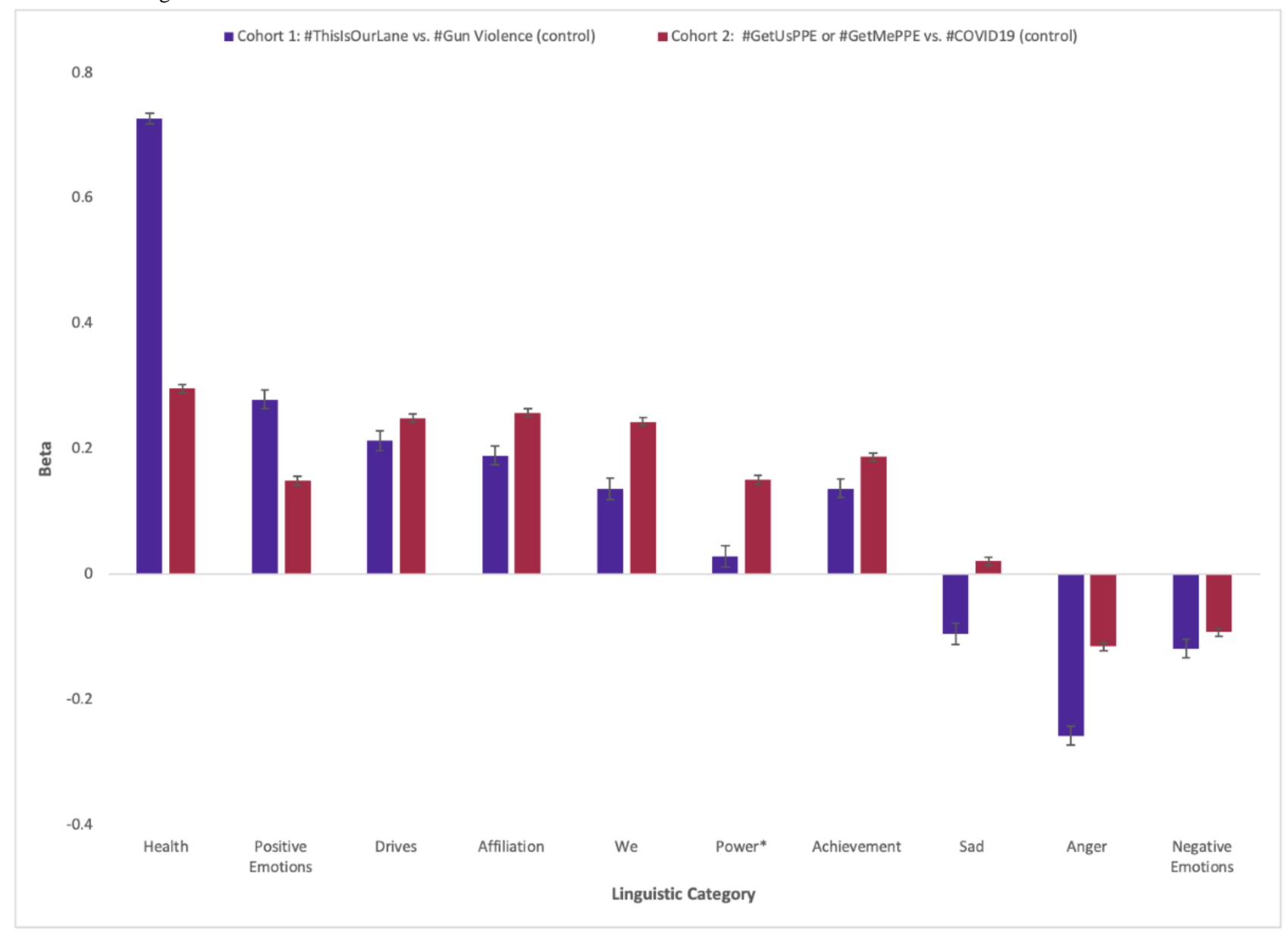

\section{Discussion}

\section{Principal Findings}

This study demonstrates not just the reach but also the inclusiveness and uniqueness of tweets containing health care-led hashtags about commonly discussed health care epidemics. Consistent with our hypotheses, tweets containing health care-led hashtags differed qualitatively and quantitatively from other tweets on the same topic during the same time period, albeit not in the way that we predicted. Tweets with \#ThisIsOurLane and \#GetUsPPE expressed more positivity and a greater sense of group affiliation than comparison hashtags led by the general public. Both \#ThisIsOurLane and \#GetUsPPE tweets contained more actionable language such as "research," "prevention," and "support."

Social media's potential as a platform for enhancing health discussions is frequently discussed $[19,20]$. Some authors have even urged the use of social media to develop grassroots "new power" movements that can combat mistruths in science and public health [21]. Others have described the potential utility of specific health care-led tweets for disseminating factual information [22]. Our analysis supports that health care-led hashtags contribute unique, actionable content and tone to national discussions about health, and can create new, inclusive movements that provide opportunities for health care professionals and non-health care-based individuals. Although we did not examine the relative prevalence of facts versus misinformation between the two sets of hashtags, the results of our study offer further evidence of the value of using Twitter to shape and build support for public health movements.

Prior literature demonstrates social media's potential for reaching new groups regarding issues in medicine and public health. However, few previous studies have characterized whether the content of social media campaigns initiated by the health care community are truly unique. For example, TikTok videos about COVID-19 accumulated over 1 billion views; however, an analysis of these videos reports that only a small portion were led by health care professionals, and that few-even those developed by the World Health Organization-included actionable tools to prevent or handle the pandemic [23,24]. Another study reported that a Twitter campaign to raise skin cancer prevention awareness led to nearly 12 million impressions on social media [25] but did not examine content or tone of shared posts. Still, others have demonstrated that health-related content on social media reflects local public health concerns and sentiments but have not examined the relative contribution of health care- versus non-health care-led hashtags [26-28]. Our work is therefore unique in examining not only the number of posts but also what differentiated them from non-health care-led posts on the same topics at the same time. 
A particularly noteworthy finding from our study is the positive tone and action-oriented content of tweets with health care-initiated hashtags. This finding differs from our expectations: we hypothesized that health care professionals would be sharing the truth about firearm injury and COVID-19, and that these realities would be negatively valenced. The finding of positive tone, even on difficult issues, may reflect societal expectations of professionalism from medical experts $[29,30]$. It may also reflect health care professionals' desire to motivate action in others: positive affect and positive tone both increase the acceptability and efficacy of behavioral interventions [31,32]. Indeed, some work has specifically provided guidance to health care and public health professionals on how to avoid or manage "trolls" [33]. Future work should examine whether successful hashtag campaigns are more positive than unsuccessful campaigns.

Establishing hashtags makes health care professionals' conversations more accessible to the nonmedical community and can be used to cultivate momentum around public health campaigns that carry educational and actionable content. Despite \#ThisIsOurLane and \#GetUsPPE being initiated and more commonly used by health care professionals, people outside of health care also commonly tweeted with these terms. Based on hashtag categories developed by Saxton et al [34], \#ThisIsOurLane and \#GetUsPPE are public education and call-to-action hashtags, which are most likely to be retweeted, and therefore most effective for online advocacy.

Future work should examine the characteristics of successful hashtag development and dissemination, as how to best create and shepherd these discussions is undetermined. Based on the origin story of \#GetUsPPE and \#ThisIsOurLane, a successful movement likely does not depend on derivation from a large company or influential organization. Instead, as Twitter increasingly serves as a news source for the general public [35], it offers a platform for average health care professionals to both spread facts and increase action on critical public health issues. Some works in the literature have developed best practices for successfully using health care hashtags to increase audience engagement [34]. Although the United States' Centers for Disease Control and Prevention has guidelines on Twitter use for health communication, initial analyses suggest mixed efficacy of their Twitter campaigns [36]. To inform others' work, future research should examine in more detail which characteristics of \#ThisIsOurLane and \#GetUsPPE enabled coalescence of a larger community.

\section{Limitations}

Limitations to this analysis include the correlational and noncausal nature of the results. This study cannot comment on whether health care-led hashtag campaigns introduced new thoughts on national health issues, as we did not review tweets from health care professionals about gun violence or the COVID-19 pandemic before the hashtags were introduced. Additionally, the magnitude of the influence of tweets with health care-led hashtags is not characterized. Finally, our analysis did not account for the voice of patients and survivors, who have previously been shown to have a powerful role on Twitter.

\section{Conclusion}

Historically, health care professionals play defining roles in social justice and public health movements. Health care-led hashtag campaigns are positive, actionable, and portray a united front in developing solutions to pressing public health issues. The \#ThisIsOurLane and \#GetUsPPE movements exemplify how online media can influence 21 st-century social dialogues about disease, injury, and prevention.

\section{Acknowledgments}

RSB and MLR are both funded by R24 HD087149 (PI: Cunningham). SCG acknowledges the support from Google Cloud.

\section{Conflicts of Interest}

AO and MLR are volunteers with the organization GetUsPPE.org. RSB reports receiving grants from the National Institute of Mental Health, National Cancer Institute, National Institute on Aging, National Heart, Lung, and Blood Institute, National Institute of Nursing Research, National Institute of Allergy and Infectious Diseases, the National Psoriasis Foundation, Veterans Affairs Quality Enhancement Research Initiative, Patient Centered Outcomes Research Institute, and the Centers for Disease Control and Prevention; royalties from Oxford University Press; served as a consultant to Camden Coalition of Healthcare Providers; and receives an honorarium from Optum Behavioral Health Clinical Scientific Advisory Council. The funding organizations listed above are not related to this article and had no bearing on its outcome.

\section{References}

1. Hughes A, Wojcik S. 10 facts about Americans and Twitter. Pew Research Center. 2019 Aug 2. URL: https://www. pewresearch.org/fact-tank/2019/08/02/10-facts-about-americans-and-twitter/ [accessed 2020-05-26]

2.@Zindoctor. If your GUNS didn't kill \& maim so many - men, women, children, of all shapes, sizes \& colors - it wouldn't be in our lane. As an \#EmergencyMedicine physician, I see and treat patients \& families directly devastated by the very reason for your existence. \#ThisISOurLane. Twitter. 2018 Nov 18. URL: https://twitter.com/Zindoctor/status/ 1060338793847418885 [accessed 2020-11-11]

3. @ choo_ek. FRONTLINE HEALTH CARE WORKERS Share a pic of the PPE you're in that you need to stay safe. Tag your congresspeople and @ VP. Use the hashtag \#GetMePPE. Twitter. 2020 Mar 17. URL: https://twitter.com/choo ek/ status/1239790569510993920 [accessed 2020-11-11] 
4. Rubin R. Physicians Are Steering the Conversation About Gun Violence. JAMA 2019 Jan 15;321(2):133-135. [doi: 10.1001/jama.2018.20385] [Medline: $\underline{\text { 30548081] }}$

5. Ranney ML, Griffeth V, Jha AK. Critical Supply Shortages - The Need for Ventilators and Personal Protective Equipment during the Covid-19 Pandemic. N Engl J Med 2020 Apr 30;382(18):e41. [doi: 10.1056/nejmp2006141]

6. Get Us PPE Mission, Vision, and Values. GetUsPPE.org. 2020. URL: https://getusppe.org/mission/ [accessed 2020-11-11]

7. Porat T, Nyrup R, Calvo RA, Paudyal P, Ford E. Public Health and Risk Communication During COVID-19-Enhancing Psychological Needs to Promote Sustainable Behavior Change. Front Public Health 2020 Oct 27;8:573397 [FREE Full text] [doi: 10.3389/fpubh.2020.573397] [Medline: 33194973]

8. Rochwerg B, Parke R, Murthy S, Fernando S, Leigh J, Marshall J, et al. Misinformation During the Coronavirus Disease 2019 Outbreak. Critical Care Explorations 2020;2(4):e0098. [doi: 10.1097/cce.0000000000000098]

9. Dlatk/TwitterMySQL. GitHub. URL: https://github.com/dlatk/TwitterMySQL [accessed 2020-11-11]

10. Guntuku SC, Sherman G, Stokes DC, Agarwal AK, Seltzer E, Merchant RM, et al. Tracking Mental Health and Symptom Mentions on Twitter During COVID-19. J Gen Intern Med 2020 Sep 07;35(9):2798-2800 [FREE Full text] [doi: 10.1007/s11606-020-05988-8] [Medline: $\underline{\text { 32638321] }}$

11. Yang Q, Tufts C, Ungar L, Guntuku S, Merchant R. To Retweet or Not to Retweet: Understanding What Features of Cardiovascular Tweets Influence Their Retransmission. J Health Commun 2018 Nov 07;23(12):1026-1035 [FREE Full text] [doi: 10.1080/10810730.2018.1540671] [Medline: 30404564]

12. Georgi S, Guntuku SC, Rahman M, Himelein-Wachowiak M, Kwarteng A, Curtis B. Twitter Corpus of the \#BlackLivesMatter Movement And Counter Protests: 2013 to 2020. Arxiv. Preprint posted online September 28, 2020. URL: https://arxiv.org/ $\underline{\mathrm{abs} / 2009.00596}$

13. Guntuku SC, Schneider R, Pelullo A, Young J, Wong V, Ungar L, et al. Studying expressions of loneliness in individuals using twitter: an observational study. BMJ Open 2019 Nov 04;9(11):e030355 [FREE Full text] [doi:

10.1136/bmjopen-2019-030355] [Medline: 31685502]

14. Guntuku SC, Ramsay JR, Merchant RM, Ungar LH. Language of ADHD in Adults on Social Media. J Atten Disord 2019 Oct 08;23(12):1475-1485. [doi: 10.1177/1087054717738083] [Medline: 29115168]

15. dlatk / happierfuntokenizing. GitHub. URL: https://github.com/dlatk/happierfuntokenizing [accessed 2020-11-11]

16. Schwartz, HA, Giorgi S, Sap M, Crutchley P, Ungar L, et al. Dlatk: Differential language analysis toolkit. 2017 Presented at: Proceedings of the 2017 Conference on Empirical Methods in Natural Language Processing: System Demonstrations; Sept 2017; Copenhagen, Denmark p. 55-60. [doi: 10.18653/v1/d17-2010]

17. Blei DM, Ng AY, Jordan MI. Latent Dirichlet Allocation. J Mach Learn Res 2003;3:993-1022 [FREE Full text]

18. Pennebaker J, Boyd R, Jordan K, Blackburn K. The Development and Psychometric Properties of LIWC2015. Austin, TX: University of Texas at Austin; 2015.

19. Choo EK, Ranney ML, Chan TM, Trueger NS, Walsh AE, Tegtmeyer K, et al. Twitter as a tool for communication and knowledge exchange in academic medicine: A guide for skeptics and novices. Med Teach 2015 May;37(5):411-416. [doi: 10.3109/0142159X.2014.993371] [Medline: 25523012]

20. Park A, Conway M. Tracking Health Related Discussions on Reddit for Public Health Applications. AMIA Annu Symp Proc 2017;2017:1362-1371 [FREE Full text] [Medline: 29854205]

21. Perera K, Timms H, Heimans J. New power versus old: to beat antivaccination campaigners we need to learn from them-an essay by Kathryn Perera, Henry Timms, and Jeremy Heimans. BMJ 2019 Nov 21;367:16447. [doi: 10.1136/bmj.16447] [Medline: 31753812]

22. Rabarison KM, Croston MA, Englar NK, Bish CL, Flynn SM, Johnson CC. Measuring Audience Engagement for Public Health Twitter Chats: Insights From \#LiveFitNOLA. JMIR Public Health Surveill 2017 Jun 08;3(2):e34 [FREE Full text] [doi: 10.2196/publichealth.7181] [Medline: 28596149]

23. Basch C, Hillyer GC, Jaime C. COVID-19 on TikTok: harnessing an emerging social media platform to convey important public health messages. Int J Adolesc Med Health 2020 Aug 10;19. [doi: 10.1515/ijamh-2020-0111] [Medline: 32776899]

24. Comp G, Dyer S, Gottlieb M. Is TikTok The Next Social Media Frontier for Medicine? AEM Education and Training 2020 Oct 21. [doi: 10.1002/aet2.10532]

25. Nguyen JL, Heckman C, Perna F. Analysis of the Twitter "Don't Fry Day" Campaign. JAMA Dermatol 2018 Aug 01;154(8):961-962 [FREE Full text] [doi: 10.1001/jamadermatol.2018.1481] [Medline: 29926080]

26. Paul M, Dredze M. You are what you tweet: Analyzing twitter for public health. 2011 Presented at: Proceedings of the Fifth International AAAI Conference on Weblogs and Social Media; July 17-21, 2011; Barcelona, Spain p. 265-272 URL: https://www.aaai.org/ocs/index.php/ICWSM/ICWSM11/paper/viewFile/2880/3264

27. Ji X, Chun SA, Wei Z, Geller J. Twitter sentiment classification for measuring public health concerns. Soc Netw Anal Min 2015 May 12;5(1):13 [FREE Full text] [doi: 10.1007/s13278-015-0253-5] [Medline: $\underline{32226558}$ ]

28. Lwin MO, Lu J, Sheldenkar A, Schulz PJ, Shin W, Gupta R, et al. Global Sentiments Surrounding the COVID-19 Pandemic on Twitter: Analysis of Twitter Trends. JMIR Public Health Surveill 2020 May 22;6(2):e19447 [FREE Full text] [doi: 10.2196/19447] [Medline: $\underline{32412418]}$

29. Regis T, Steiner MJ, Ford CA, Byerley JS. Professionalism expectations seen through the eyes of resident physicians and patient families. Pediatrics 2011 Feb 17;127(2):317-324. [doi: 10.1542/peds.2010-2472] [Medline: 21242219] 
30. Sawicki NN. Judging doctors-the person and the professional. Virtual Mentor 2011 Oct 01;13(10):718-722 [FREE Full text] [doi: 10.1001/virtualmentor.2011.13.10.msoc1-1110] [Medline: 23137893]

31. Lawton R, Conner M, McEachan R. Desire or reason: predicting health behaviors from affective and cognitive attitudes. Health Psychol 2009 Jan;28(1):56-65. [doi: 10.1037/a0013424] [Medline: 19210018]

32. Muench F, van Stolk-Cooke K, Morgenstern J, Kuerbis AN, Markle K. Understanding messaging preferences to inform development of mobile goal-directed behavioral interventions. J Med Internet Res 2014 Feb 05;16(2):e14 [FREE Full text] [doi: 10.2196/jmir.2945] [Medline: 24500775]

33. Jamison AM, Broniatowski DA, Quinn SC. Malicious Actors on Twitter: A Guide for Public Health Researchers. Am J Public Health 2019 May;109(5):688-692. [doi: 10.2105/AJPH.2019.304969] [Medline: 30896994$]$

34. Saxton GD, Niyirora JN, Guo C, Waters RD. \#AdvocatingForChange: The Strategic Use of Hashtags in Social Media Advocacy. ASW 2015 Jul 27;16(1):154-169. [doi: 10.18060/17952]

35. News use across social media platforms 2018. Pew Research Center. 2018 Sep 10. URL: https://www.journalism.org/2018/ 09/10/news-use-across-social-media-platforms-2018/ [accessed 2020-11-11]

36. Chen S, Xu Q, Buchenberger J, Bagavathi A, Fair G, Shaikh S, et al. Dynamics of Health Agency Response and Public Engagement in Public Health Emergency: A Case Study of CDC Tweeting Patterns During the 2016 Zika Epidemic. JMIR Public Health Surveill 2018 Nov 22;4(4):e10827 [FREE Full text] [doi: 10.2196/10827] [Medline: 30467106]

\section{Abbreviations}

API: application programming interface

MALLET: Machine Learning for Language Toolkit

PPE: personal protective equipment

Edited by G Eysenbach; submitted 28.09.20; peer-reviewed by T Chan, K Utter; comments to author 23.10.20; revised version received
12.11.20; accepted 12.12.20; published 06.01.21
Please cite as:
Ojo A, Guntuku SC, Zheng M, Beidas RS, Ranney ML
How Health Care Workers Wield Influence Through Twitter Hashtags: Retrospective Cross-sectional Study of the Gun Violence and
COVID-19 Public Health Crises
JMIR Public Health Surveill $2021 ; 7(1): e 24562$
URL: $\underline{\text { https://publichealth.jmir.org/2021/1/e } 24562}$
doi: $\underline{10.2196 / 24562}$
PMID: $\underline{3315578}$

(C)Ayotomiwa Ojo, Sharath Chandra Guntuku, Margaret Zheng, Rinad S Beidas, Megan L Ranney. Originally published in JMIR Public Health and Surveillance (http://publichealth.jmir.org), 06.01.2021. This is an open-access article distributed under the terms of the Creative Commons Attribution License (https://creativecommons.org/licenses/by/4.0/), which permits unrestricted use, distribution, and reproduction in any medium, provided the original work, first published in JMIR Public Health and Surveillance, is properly cited. The complete bibliographic information, a link to the original publication on http://publichealth.jmir.org, as well as this copyright and license information must be included. 